BREAST CANCER

\title{
A rude awakening from tumour cells
}

\begin{abstract}
In women who have had breast cancer, drug treatments are often stopped five years after removal of the primary tumour. A meta-analysis shows that these individuals are still at risk of relapse.
\end{abstract}

\section{GIUSEPPE CURIGLIANO \& FATIMA CARDOSO}

$\mathrm{T}$ The main aim of adjuvant therapy, which is given after an apparently successful primary cancer treatment, is to reduce the risk of local and distant metastatic disease relapse owing to residual breast-tumour cells that can persist for years or decades in a dormant state. Our knowledge of the biology of dormant residual disease is cripplingly limited. Writing in the New England Journal of Medicine, Pan et al. ${ }^{1}$ examine rates of metastatic cancer spread in 62,923 women treated for breast cancer and given adjuvant therapy. The authors' findings provide a window on dormancy in this disease.

Pan and colleagues performed a metaanalysis of 88 trials involving women who had ER-positive breast cancers - subtypes of breast cancer characterized by expression of the oestrogen receptor (ER). The women were all disease-free after five years of scheduled adjuvant endocrine therapy, which involves taking drugs that lower the activity of the ER. The beneficial effects of these treatments for preventing metastasis during the 5 years following diagnosis are not in doubt - instead, the authors' analysis was designed to determine the risk of late metastasis occurring between years 5 and 20, if adjuvant therapy is stopped after 5 years. They found that metastasis occurred at a steady rate for the 15 years after the end of the treatment period.

Remarkably, the most powerful determinants of the risk of recurrence were those originally used to grade the aggressiveness of the primary cancer - the diameter of the tumour and the number of lymph nodes containing cancerous cells, which indicates whether the primary disease had spread at the start of treatment. However, even among women with small, node-negative tumours, the risk of metastasis was about $10 \%$ over the 15 -year period.

Pan et al. acknowledge that there are several caveats to their analysis, including a lack of available data on how many women completed their adjuvant treatment, and suboptimal treatment of people who had one particular type of ER-positive breast cancer, dubbed HER2-positive. Nonetheless, it is clear that, even after adjuvant endocrine therapy, women with ER-positive, early-stage breast cancer remain at persistent risk of recurrence for at least 20 years after the original diagnosis. These findings, together with data from another analysis of breast-cancer relapse ${ }^{2}$, might have implications for long-term followup strategies, and possibly for treatments.

One way to decrease the risk of relapse might be to extend the duration of adjuvant endocrine therapy - a strategy that is already being tested. We wonder, however, whether this approach will be sufficient to reduce or avoid the risk of late metastasis. One trial ${ }^{3}$ published in 2016 indicates that increasing the duration of adjuvant therapy from five to ten years significantly improves rates of diseasefree survival five years after the treatment
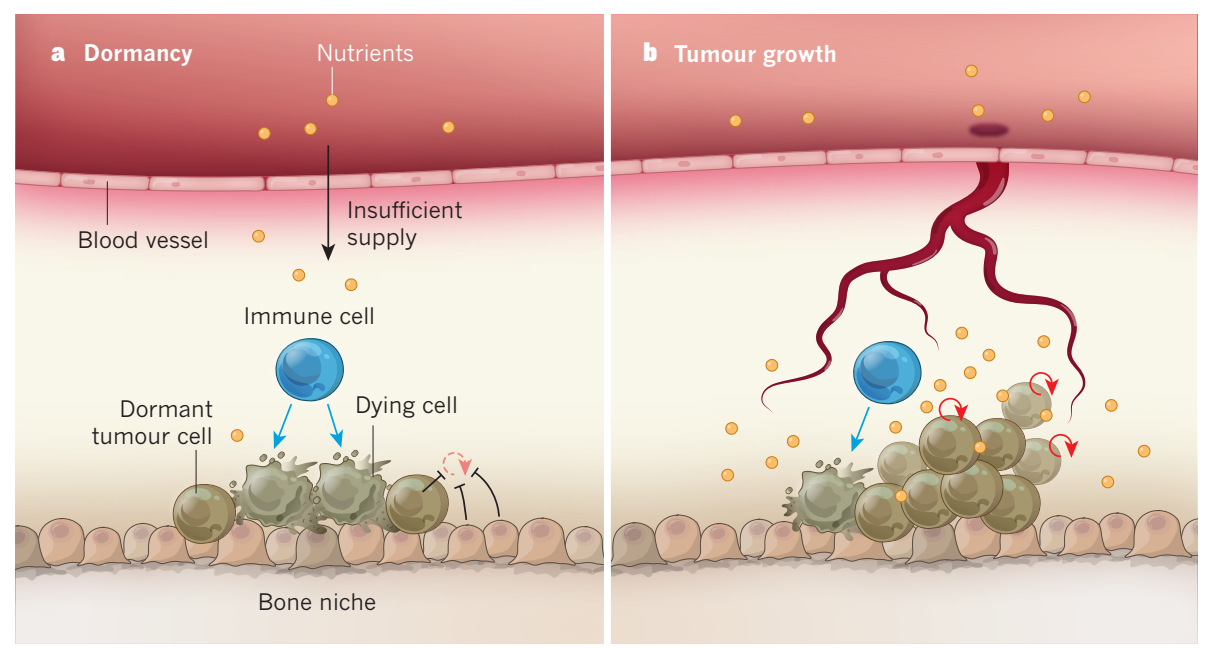

Figure 1 | Multiple mechanisms underlying tumour dormancy. Pan et al. ${ }^{1}$ report that women who take drugs for five years after their primary breast cancer has been removed are still at risk of a late relapse, because of tumour cells that have migrated to a secondary site such as the bone and adopted a dormant state. a, Tumour-cell dormancy can involve many factors, including: poor blood-vessel supply, which means that there is insufficient oxygen and nutrients for proliferation; ongoing surveillance by immune cells that kill some dormant tumour cells; and signals, both tumour-cell-intrinsic and from cells in the surrounding bone niche, that inhibit proliferation (dashed curly arrow). b, Relapse occurs when the balance between proliferation and these factors is lost, because of vessel growth, evasion of immune-cell activity or changes that prevent proliferation-blocking signals, leading to tumour growth. destruction by the body's immune system ceases, and lowers the incidence of cancer arising in the previously unaffected breast but does not increase overall survival rates. A second ${ }^{4}$ trial compared continuous adjuvant therapy between years five and ten with an 'on-off' therapy that aimed to resensitize cancer cells that might have become resistant therapy. It found no difference in rates of metastasis-free survival on completion of either treatment.

Longer follow-ups are needed to better understand the effects of extended adjuvant endocrine therapy, because of the slowly progressive nature of the disease. Nonetheless, it is clear that, although extending the duration of therapy can play a part in preventing late relapses, it might well need to be given for the rest of a woman's life to be effective. This would raise issues of toxicity, compliance and cost.

To best determine other possible ways to reduce the risk of relapse, we must consider might cause the dormant tumour cells from which metastasis arises to reawaken and entered a microenvironment that supports their survival ${ }^{5}$. Once in this niche, many mechanisms might underlie dormancy indeed, dormancy can be thought of as a multidimensional status, which involves mulple factors (Fig. 1)

One dimension is cellular dormancy, in which intrinsic or extrinsic factors drive cells into a resting state. Intrinsic factors might 
include changes that reduce the cell's drive to divide, similar to those that limit proliferation in cancer stem cells (for example, see ref. 6). These changes could be epigenetic - modulating gene expression without affecting the underlying DNA sequence - or genetic ${ }^{5}$. Extrinsic factors include crosstalk between different cell types in the surrounding microenvironment, such as endothelial cells that line vessels, immune cells and fibroblasts, which make up the structural framework of tissues. A second dimension is vasculature-related dormancy, in which the tumour-cell population is kept small because poor vascularization in the region leads to a lack of nutrients and oxygen. A third dimension is immune-mediated dormancy, in which the immune system controls an expanding tumour-cell population by continuously searching for and eliminating cancerous cells.

If this dormancy status becomes unbalanced owing to changes in any of these factors, dormant cells awaken and metastatic disease develops. Thus, strategies to prevent relapse should aim either to stop dormant cells from awakening or to destroy them when dormant.
To achieve this goal, it would be beneficial to identify those patients who will have a late relapse and to tailor a therapeutic strategy to them. ER-positive breast cancers have a low level of intratumoral cell diversity ${ }^{7}$, such as distinct forms of genetic, epigenetic and functional diversity. A better understanding of this diversity might lead to the identification of factors that enable certain cells to become resistant to endocrine therapy and survive in metastatic niches.

Perhaps the major effect of extended adjuvant endocrine therapy is to keep tumour cells dormant for a longer, but still temporary, period of time. We believe that a combined strategy that concomitantly targets both the tumour cells and the surrounding microenvironment has a higher probability of destroying the dormant cells or of inducing a lifelong state of dormancy, and hence provides a higher probability of a cure. A better understanding of the crosstalk between the dormant cells, their surrounding cell types and the immune system is crucial for developing effective microenvironment-directed therapies. In addition, attention should be paid to the possibility that dormant breast tumour cells undergo evolutionarily conserved programs that lead to a stem-cell-like, prolonged resting state. Maintaining this stem-cell status permanently might be another way to prevent dormant cells from reawakening.

Giuseppe Curigliano is in the Department of Hematology and Oncology, European Institute of Oncology, University of Milan, 20141 Milan, Italy. Fatima Cardoso is at the Breast Unit, Champalimaud Clinical Center, Champalimaud Foundation, 1400-038 Lisbon, Portugal.

e-mails: giuseppe.curigliano@ieo.it; fatimacardoso@fundacaochampalimaud.pt

1. Pan, H. et al. N. Engl. J. Med. 377, 1836-1846 (2017).

2. Colleoni, M. et al. J. Clin. Oncol. 34, 927-935 (2016).

3. Goss, P. E. et al. N. Engl. J. Med. 375, 209-219 (2016).

4. Colleoni, M. et al. Lancet Oncol. 19, 127-138 (2017).

5. Goss, P. E. \& Chambers, A. F. Nature Rev. Cancer 10, 871-877 (2010).

6. Tosoni, D. et al. EMBO Mol. Med. 9, 655-671 (2017).

7. Ellis, M. J. \& Perou, C. M. Cancer Discov. 3, 27-34 (2013). 